\title{
Une intoxication volontaire grave à la venlafaxine
}

\section{Acute non-fatal venlafaxine self-poisoning : \\ a case report}

\section{Guillaume HOIZEY $^{(1) *}$, Francis GROSSENBACHER ${ }^{(2)}$, Aurélie MOLIA ${ }^{(3)}$, Laurence BAGREL ${ }^{(3)}$, Monique CARLIER ${ }^{(2)}$, Thierry TRENQUE( ${ }^{(3)}$, Hervé MILLART ${ }^{(1)}$, Denis LAMIABLE ${ }^{(1)}$}

(1) Laboratoire de Pharmacologie et Toxicologie, Hôpital Maison-Blanche, CHU de REIMS - FRANCE (2) Service d'Aide Médicale Urgente, Centre de Toxicovigilance, CHU de REIMS - FRANCE (3) Centre Régional de Pharmacovigilance, CHU de REIMS - FRANCE

* Auteur à qui adresser la correspondance : Guillaume HOIZEY, Laboratoire de Pharmacologie et Toxicologie, CHU de Reims, 45, rue Cognacq-Jay - 51092 REIMS CEDEX - FRANCE Tél : (33) 0326787530 - Fax : (33) 0326788456 - E-mail : ghoizey@chu-reims.fr

Ce travail a été présenté au XI⿳亠ेme congrès de la Société Française de Toxicologie Analytique, 11-13 juin 2003, DINARD-FRANCE

(Reçu le 7 octobre 2003 ; accepté le 22 décembre 2003)

\section{$R \hat{E} S U M E ́$}

Une jeune femme de 24 ans est admise aux urgences dans un état de coma réactif, secondaire à l'ingestion volontaire, probable, de 60 comprimés de venlafaxine dosés à $50 \mathrm{mg}$. Durant son transport et à son arrivée à l'hôpital, elle présente plusieurs crises convulsives généralisées. L'examen clinique montre une tachycardie, avec à l'ECG un rythme sinusal à QRS fins. L'interrogatoire révèle l'absorption en une prise unique, environ 2 heures précédant l'admission aux urgences, des 3 grammes de venlafaxine, sans aucune autre substance associée. Après une prise en charge thérapeutique conventionnelle avec administration de clonazé. pam, la patiente quitte le service de réanimation 48 heures après son arrivée. Aucune séquelle n'est à déplorer. Les analyses toxicologiques effectuées sur les tout premiers prélèvements sanguins ont permis de confirmer, au moyen de la chromatographie en phase gazeuse couplée à la spectrométrie de masse, la nature du composé incriminé. Plusieurs prélèvements consécutifs ont été réalisés pendant les 24 pre-

\section{SUMMARY}

A 24 year-old young women was admitted to emergency unit, after the ingestion of 60 venlafaxine $50-\mathrm{mg}$ tablets. During transport and after her arrival at the hospital, she experienced several generalized seizures. Physical examination. revealed a coma, and the electrocardiogram showed a sinus tachycardia with a normal $Q R S$ complex. On further questioning, she stated that she had ingested $3 \mathrm{~g}$ of venlafaxine, approximately 2 hours prior to her admission. She denied ingesting any other medication. Following a classical and appropriate treatment, she recovered completely and was discharged after 48 hours. Initial toxicological testing permitted us to confirm the overdose by that compound. Venlafaxine was identified in plasma by gas-chromatography and mass-spectrometry detection. Blood specimens were serially collected from her admission up to 24 hours after poisoning. Concentrations of venlafaxine and its major metabolite, $O$-desmethylvenlafaxine, were determined in plasma by using a high-performance liquid chromatography 
mières heures de surveillance en service de réanimation. Les concentrations plasmatiques de venlafaxine et de son principal métabolite actif, l'O-déméthylvenlafaxine, ont été déterminées par chromatographie liquide haute performance et détection ultraviolette. Ce cas d'intoxication avec 3 grammes de venlafaxine absorbée isolément, documenté par des dosages sanguins répétés, n'a, à notre connaissance, pas encore été décrit en France. Sur le plan de la symptomatologie clinique et du suivi toxicocinétique, cette observation est en accord avec plusieurs données de la littérature. Enfin, il apparaît que la persistance d'une tachycardie sinusale au moins pendant 12 heures suivant l'intoxication, soit en relation avec le maintien, sur cette même période, de concentra. tions de venlafaxine et de son métabolite actif, supérieures aux concentrations thérapeutiques.

\section{MOTS-CLES}

Venlafaxine, intoxication aiguë, toxicocinétique.

\section{Introduction}

La venlafaxine est un antidépresseur de la famille des inhibiteurs de la recapture de la sérotonine et de la noradrénaline (IRSNA), et dans une moindre mesure, de la dopamine. Elle ne présente quasiment pas d'affinité avec les récepteurs muscariniques, histaminiques et adrénergiques, lui conférant ainsi un bon profil de tolérance (1). Par métabolisme hépatique, via les enzymes du cytochrome P450 2D6, la venlafaxine est transformée en un métabolite actif, l'O-déméthylvenlafaxine. Commercialisée en France sous le nom d'Effexor ${ }^{\circledR}$ (Wyeth-Lederlé, Puteaux, France) en conditionnement de 30 comprimés dosés à 25 ou $50 \mathrm{mg}$, la venlafaxine est utilisée habituellement à des posologies de $75 \mathrm{mg}$ par jour, pouvant être augmentées, dans la prise en charge des dépressions sévères, jusqu'à une posologie quotidienne maximale de $375 \mathrm{mg}$ (2). Il existe également une forme à libération prolongée.

A ce jour, plusieurs cas d'intoxications aiguës à la venlafaxine ont été rapportés, le plus souvent dans un contexte d'intoxication volontaire poly-médicamenteuse. Les principaux signes cliniques décrits lors d'une l'intoxication sévère sont, une profonde dépression du système nerveux central (3), avec survenue de convulsions ou encore d'un syndrome sérotoninergique (4), et, plus rarement, l'apparition de troubles cardiovasculaires (5). Bien que plusieurs auteurs documentent leurs observations cliniques au moyen de dosages sanguins isolés $(3,6)$, peu de données toxicocinétiques sur la venlafaxine sont disponibles suite à une intoxication aiguë $(5,7)$.

Nous rapportons un cas d'intoxication volontaire non mortel, secondaire à l'ingestion d'une dose massive en une prise unique de venlafaxine seule. L'évolution au plan clinique est décrite parallèlement au devenir, durant les 24 heures suivant l'intoxication, des concen- assay. Following a basic toxicokinetics study, the results are discussed in light of both literature, and development of clinical symptoms.

\author{
KEY-WORD \\ Venlafaxine, acute poisoning, toxicokinetics.
}

trations plasmatiques de la venlafaxine et de son principal métabolite actif, l' O-déméthylvenlafaxine.

\section{Observation clinique}

Une jeune femme de 24 ans présentant un coma réactif (score de Glasgow $=8$ ), est admise dans le service des urgences d'un hôpital périphérique au CHU de Reims, suite à l'absorption très probable de 2 boîtes de 30 comprimés d'Effexor ${ }^{\circledR}$ dosés à $50 \mathrm{mg}$, soit $3 \mathrm{~g}$ au total. Durant son transport et lors de son admission, elle présente 2 crises convulsives généralisées, nécessitant une prise en charge conventionnelle avec notamment l'administration de clonazépam (1 ampoule de Rivotril ${ }^{\circledR}$ injectable dosée à $1 \mathrm{mg}$ ). L'examen clinique ne montre pas d'hyperthermie, de sueurs, de raideur de nuque ou encore d'éruption cutanée. Les pupilles sont en mydriase réactive. La saturation en oxygène $\left(\mathrm{SaO}_{2}\right)$ est mesurée à $99 \%$ en air ambiant. Au plan cardiovasculaire, la pression artérielle est chiffrée à 100/70 $\mathrm{mmHg}$, avec secondairement un épisode à $80 / 50 \mathrm{mmHg}$, conduisant à un remplissage vasculaire par $500 \mathrm{ml}$ d'un succédané du plasma (Elohes $®$ ). L'ECG montre une tachycardie sinusale (160 pulsations/min) avec des complexes QRS fins (QRS $<0,10$ seconde). L'interrogatoire de la patiente, réalisé à son réveil, a permis de confirmer la prise à visée suicidaire, dans les 2 heures précédant son admission à l'hôpital, de $3 \mathrm{~g}$ de venlafaxine en une prise unique, et sans aucune autre substance médicamenteuse associée. Le traitement a consisté en une épuration digestive par administration initiale de $50 \mathrm{~g}$ de charbon activé (Carbomix $®$ ). La patiente se plaint alors d'une toux importante. C'est à ce moment précis qu'une nouvelle crise convulsive survient, qui sera suivie quasi-immédiatement par une polypnée, avec chute de la $\mathrm{SaO}_{2}$ en dessous de $90 \%$, nécessitant l'administration d'oxygène. L'hypothèse d'une inhalation bron- 
chique sera rapidement confirmée par la radiographie pulmonaire. La dégradation de la fonction ventilatoire (pO2 à $57 \mathrm{mmHg}$ et $\mathrm{SaO}_{2}$ à $89 \%$, sous $6 \mathrm{l} / \mathrm{min}$ d'oxygène) motive le transfert de la patiente vers le $\mathrm{CHU}$ de Reims.

A son arrivée en service de réanimation, la priorité est donnée à la restauration de la fonction respiratoire. $\mathbf{L a}$ surveillance dans ce service permettra d'objectiver la persistance d'une tachycardie sinusale régulière (150 pulsations/min) pendant une durée d'environ 12 heures, sans chute tensionelle, suivie du maintien d'une fréquence cardiaque à 110 pulsations/min, les 12 heures suivantes. L'évolution est favorable après $48 \mathrm{~h}$, avec notamment une fonction hémodynamique stable, un état de conscience tout à fait normal, et l'absence de récidive des crises convulsives. Durant l'hospitalisation, quatre échantillons sanguins ont pu été prélevés au temps 2, 5, 15 et 25 heures suivant l'intoxication, en vue d'analyses toxicologiques.

\section{Matériels et méthodes}

\section{A la prise en charge initiale}

A la prise en charge initiale de la patiente, l'éthanolémie et le dosage de méprobamate étaient réalisés par chromatographie en phase gazeuse avec détection par ionisation de flamme (CPG-FID). Le dépistage de molécules stupéfiantes (opiacés, cocaïne, cannabis et amphétamines) dans les urines était effectué par méthode immunochromatographique. Les substances psychotropes appartenant aux classes médicamenteuses suivantes, benzodiazépines, barbituriques et antidépresseurs tricycliques étaient recherchées par immunoanalyse.

Sur l'échantillon sanguin prélevé dès les premiers instants de l'hospitalisation, une recherche complémentaire, plus étendue, a pu être réalisée selon notre procédure de screening toxicologique, au moyen du couplage CPG et spectrométrie de masse (CPG-SM), après extraction liquide-liquide de l'échantillon par l'éther en milieu alcalin.

\section{Suivi toxicocinétique de la venlafaxine et de la O-déméthylvenlafaxine}

La disponibilité de plusieurs prélèvements sanguins sur les 24 heures suivant l'intoxication, a permis d'envisager le suivi toxicocinétique de la venlafaxine et de son métabolite actif.

L'analyse plasmatique de ces deux molécules est réalisée en chromatographie en phase liquide et détection ultraviolette selon une adaptation de la méthode décrite par Matoga et coll. (8). Après une extraction liquide- liquide (hexane/alcool isoamylique $98 / 2 ; \mathrm{v} / \mathrm{v}$ ) en milieu alcalin, la venlafaxine et son métabolite sont chromatographiés en phase inverse sur une colonne de type C8 (Lichrospher® 100CH8 - 5 $\mathrm{mm}-240 \times 4,6 \mathrm{~mm}$ - Merck). La phase mobile, délivrée à un débit de 1,2 $\mathrm{ml} / \mathrm{min}$, est composée du mélange isocratique acétonitrile/tampon phosphate ajusté à pH3 $(32 / 68 ; \mathrm{v} / \mathrm{v})$. La détection est effectuée à $229 \mathrm{~nm}$ et le para-méthyl milnacipran est utilisé comme étalon interne. Un chromatogramme d'un échantillon plasmatique surchargé à $500 \mu \mathrm{g} / \mathrm{l}$ de venlafaxine et d'O-déméthylvenlafaxine est présenté en figure 1.

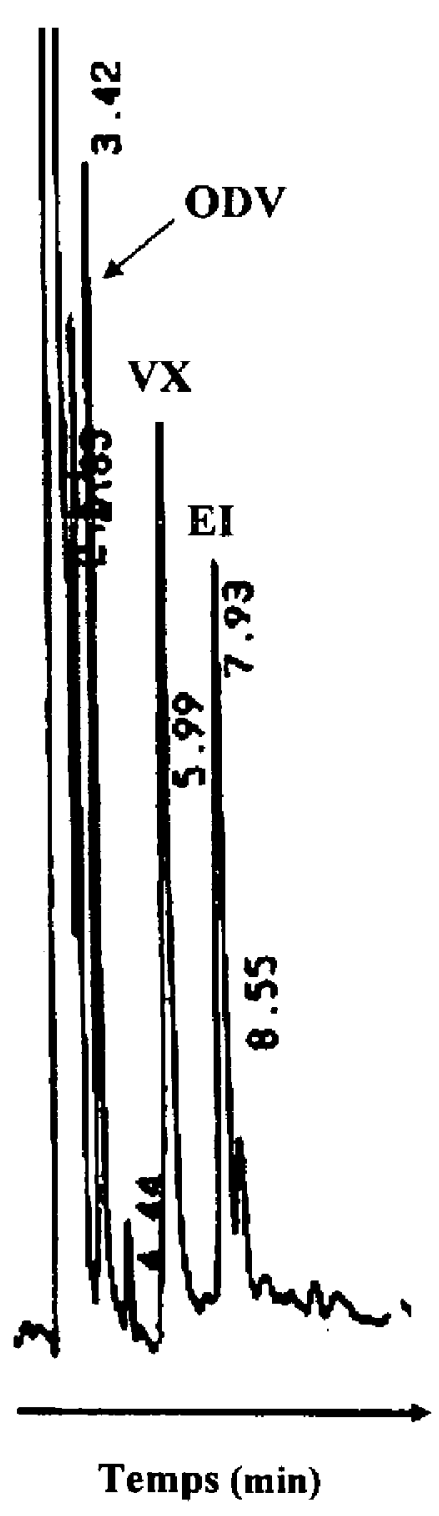

Figure 1 : Chromatogramme d'un extrait plasmatique contenant de la venlafaxine (VX), de l'O-desméthylvenlafaxine $(O D V)$ et l'étalon interne (EI), le para-méthyl milnacipran. La concentration de VX et d'ODV est de $500 \mu \mathrm{g} / \mathrm{l}$.

\section{Résultats}

\section{A la prise en charge initiale}

L'éthanolémie était inférieure à $0,1 \mathrm{~g} / \mathrm{l}$. Le dépistage urinaire de molécules stupéfiantes ainsi que la recherche de substances psychotropes dans le sang se sont révélés négatifs. Le dosage de méprobamate montrait une concentration plasmatique inférieure à $0,5 \mathrm{~g} / \mathrm{l}$. 
L'analyse complémentaire mise en œuvre par CPG-SM a permis de confirmer la présence de venlafaxine dans l'échantillon sanguin. Dans nos conditions opératoires, aucun autre xénobiotique n'a pu être identifié.

\section{Suivi toxicocinétique de la venlafaxine et de la O-déméthylvenlafaxine}

La figure 2 représente l'évolution des concentrations plasmatiques de la venlafaxine et de l'O-déméthylvenlafaxine en fonction du temps. Ces profils sont caractérisés par une phase initiale au cours de laquelle les concentrations maximales plasmatiques des deux composés sont obtenues environ 5 heures suivant l'ingestion d'Effexor@ (respectivement $12190 \mu \mathrm{g} / \mathrm{l}$ et 1874 $\mu \mathrm{g} /$ pour la venlafaxine et son métabolite). Elle est suivie d'une phase de décroissance de ces concentrations, qui atteignent à la $25^{\text {eme }}$ heure des valeurs de $627 \mu \mathrm{g} / \mathrm{l}$ pour la venlafaxine et $381 \mu \mathrm{g} / \mathrm{l}$ pour l'O-déméthylvenlafaxine. Bien que le faible nombre de mesures effectuées sur 24 heures ne permette pas une analyse toxicocinétique approfondie, il est toutefois possible d'évaluer graphiquement les demi-vies des phases terminales de chacune de ces deux molécules. C'est ainsi que la venlafaxine et son métabolite actif présentent des demi-vies plasmatiques d'élimination estimées, respectivement à 4,8 heures et 7,2 heures.

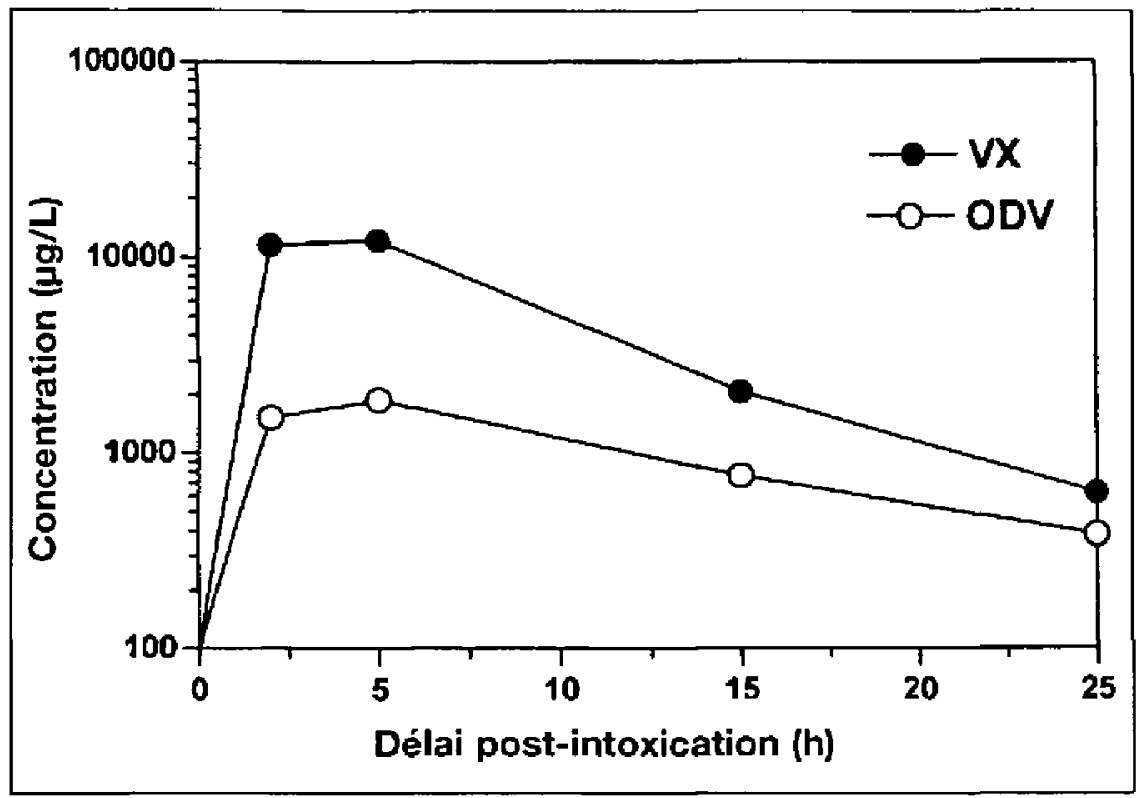

Figure 2 : Évolution des concentrations plasmatiques de la venlafaxine (VX) et de l'O-desméthylvenlafaxine (ODV) en fonction du temps.

\section{Discussion}

Si de nombreux cas d'intoxications aiguës volontaires par la venlafaxine ont été décrits pour des doses ingérées très importantes, le plus souvent supérieures à $2 \mathrm{~g}$ $(3,5,7,9)$, il s'agit, à notre connaissance, du premier cas rapporté en France, d'intoxication massive avec $3 \mathrm{~g}$ de venlafaxine, absorbée isolément. Au plan clinique, les principales manifestations observées, notamment la survenue de convulsions généralisées et d'une tachycardie sinusale, sont tout à fait en accord avec les données de la littérature pour des doses ingérées analogues $(5,9,10)$. L'administration de clonazépam a fait céder les crises convulsives et probablement limité les autres manifestations neurologiques en rapport avec l'intoxication.

Par ailleurs, il convient de rappeler qu'un surdosage en venlafaxine est susceptible, à l'instar des substances médicamenteuses de la classe thérapeutique des IRSNA, de donner lieu à un syndrome sérotoninergique, le plus souvent lors d'associations à d'autres molécules pro-sérotoninergiques (11). Dans notre cas, l'absence de critères cliniques différentiels tels que hyperthermie, sueurs profuses ou incoordination des mouvements $(11,12)$ ne permettait pas de conclure à ce diagnostic. Enfin, il ressort des données de la littérature que les cas de syndrome sérotoninergique secondaire à l'absorption massive et isolée de venlafaxine sont rares. A notre connaissance, seules deux observations ont été publiées à ce jour $(4,6)$.

Les concentrations plasmatiques de venlafaxine et d'Odéméthylvenlafaxine, retrouvées 2 heures et 5 heures après la prise, ont permis de confirmer le diagnostic d'intoxication aiguë par cette molécule. Les valeurs retrouvées sont très largement supérieures aux concentrations thérapeutiques habituelles, ces dernières étant de l'ordre de 100 à $200 \mu \mathrm{g} / \mathrm{l}$ pour la venlafaxine seule, et de 250 à $750 \mu \mathrm{g} / \mathrm{l}$ lorsque la concentration du métabolite actif est également prise en compte (11). Les concentrations observées chez cette patiente sont assez comparables à celles rapportées dans plusieurs cas d'intoxications sévères $(3,7,8)$, et même lors de décès imputés à la prise de venlafaxine $(13,14,15)$. C'est ainsi que dans un cas post-mortem, Labat et coll. rapportent des concentrations sanguines de venlafaxine et de son métabolite respectivement de $4900 \mu \mathrm{g} / 1$ et 5200 $\mu \mathrm{g} / \mathrm{l}$ (13). De même, dans l'étude de Goehringer et coll., les concentrations sanguines post-mortem de venlafaxine et d'O-déméthylvenlafaxine retrouvées chez 4 personnes dont le décès pouvait être relié à l'ingestion massive de venlafaxine, variaient de 1800 à $36000 \mu \mathrm{g} / \mathrm{L}$ pour la venlafaxine, et de 700 à $3500 \mu \mathrm{g} / \mathrm{L}(\mathrm{n}=3)$ pour son métabolite (15).

Dans notre observation, le manque de données n'autorise évidemment pas une analyse fine du suivi toxicocinétique de la venlafaxine et de son métabolite. Toutefois, les demi-vies plasmatiques estimées pour ces deux molécules (environ 5 et 7 heures, respectivement pour la venlafaxine et son métabolite O-déméthylé) sont proches de celles déterminées dans un contexte d'utilisation thérapeutique, à savoir environ 5 heures pour la venlafaxine et 11 heures pour l'O-déméthyl- 
venlafaxine (16). Récemment, Langford et coll. (7) ont rapporté un cas similaire d'intoxication, avec 3 grammes de venlafaxine. Le suivi régulier des concentrations plasmatiques sur 2 jours a permis de mettre en évidence des résultats différents des nôtres, à savoir une concentration maximale atteinte tardivement après I'ingestion (près de 10 heures), et des demi-vies d'éliminations, augmentées jusqu'à 15 heures pour la venlafaxine. Sur ce dernier point, les auteurs suggèrent, du fait de l'existence d'un polymorphisme génétique sur le cyctochrome P450 2D6 (isoenzyme impliquée dans le métabolisme de cette molécule), la possibilité que certains patients présentent le phénotype métaboliseur lent, se traduisant ainsi par une vitesse de métabolisation plus lente. Cette hypothèse, que Blythe et coll. avaient déjà évoquée dans un cas semblable d'intoxication à la venlafaxine (5), tend à démontrer que l'intensité et la durée des manifestations cliniques liées à l'intoxication par la venlafaxine pourraient également être dépendantes des caractéristiques génotypiques propres au patient.

Enfin, il est intéressant de constater que, dans notre cas, la persistance d'une tachycardie sinusale pendant au moins les 12 premières heures suivant l'intoxication, peut être mise en relation avec le maintien, sur cette même période, de concentrations de venlafaxine et d'O-déméthylvenlafaxine très nettement supérieures aux concentrations thérapeutiques. De même, la diminution de la fréquence cardiaque lors des 12 heures suivantes semble bien corrélée à la décroissance des concentrations plasmatiques.

\section{Conclusion}

Cette observation d'intoxication volontaire à la venlafaxine illustre, de nouveau, la capacité de cette molécule à provoquer, dans un contexte de prise massive et isolée, des symptômes neurologiques graves à type de convulsions, associés à une tachycardie sinusale. L'analyse toxicologique était utile pour confirmer l'origine de l'intoxication aiguë. Des dosages réitérés de la venlafaxine et de son principal métabolite actif pendant 24 heures, ont permis, en dépit de leur faible nombre, d'envisager un suivi toxicocinétique.

\section{Références}

1. Holliday S.M., Benfield P. Venlafaxine : a review of its pharmacology and therapeutic potential. Drugs 1995 ; $49: 280-94$.

2. Dictionnaire VIDALß $2003-79^{\mathrm{eme}}$ Edition. Paris France.

3. Fantaskey A., Burkhart K.K. A case report of venlafaxine toxicity. J. Toxicol. Clin. Toxicol. $1995 ; 33(4)$ : 35961.

4. Daniels R.J. Serotonin syndrome due to venlafaxine overdose. J. Accid. Emerg. Med. 1998 ; 15 : 333-7.

5. Blythe D., Hackett L.P. Cardiovascular and neurological toxicity of venlafaxine. Hum. Exp. Toxicol. $1999 ; 18$ : 309-13.

6. Kolecki P. Isolated venlafaxine-induced serotonin syndrome. J. Emerg. Med. 1997 ; 15(4) : 491-3.

7. Langford N.J., Martin U., Ruprah M., Ferner R.E. Alternative venlafaxine kinetics in overdose. J. Clin. Pharm. Therap. $2002 ; 27: 465-7$.

8. Matoga M., Pehourcq F., Titier K., Dumora F., Jarry C. Rapid high-performance liquid chromatographic measurement of venlafaxine and O-desmethylvenlafaxine in human plasma. Application to management of acute intoxications. J. Chromatogr. B. 2001 ; $760: 213-8$.

9. White C.M., Gailey R.A., Levin G.M., Smith T. Seizure resulting from a venlafaxine overdose. Ann. Pharmacother. 1997 ; $31: 178-80$.

10. Leaf E.V. Comment: venlafaxine overdose and seizure. Ann. Pharmacother. ; 1998 ; 32 : 135-6.

11. Kergueris M.F., Danel V. Syndrome sérotoninergique Approche analytique en toxicologie hospitalière. Ann. Toxicol. Anal. $2002 ; 14(2): 105-10$.

12. Sternbach $\mathbf{H}$. The serotonin syndrome. Am. J. Psychiatry $1991 ; 148: 705-13$.

13. Labat L., Deveaux M., Dallet P., Dubost J.P. Separation of new antidepressants and their metabolites by micellar electrokinetic capillary chromatography. J. Chromatogr. B. ; $2002 ; 773: 17-23$.

14. Parsons A.T., Anthony R.M., Meeker J.E. Two fatal cases of venlafaxine poisoning. J. Anal. Toxicol. $1996 ; 20$ : 266-8.

15. Goeringer K.E., Mc Intyre I.M., Drummer O.H. Postmortem tissue concentrations of venlafaxine. Forensic Sci. Int. 2001 ; 121(1-2) : 70-5.

16. Klamerus K.J., Maloney K., Rudolph R.L., Sisenwine S.F., Jusko W.J., Chiang S.T. Introduction of a composite parameter to the pharmacokinetics of venlafaxine and its active O-desmethyl metabolite. J. Clin. Pharmacol. $1992 ; 32: 716-24$. 